Pacific

Journal of

Mathematics

THE CLASSIFICATION OF COHOMOLOGY ENDOMORPHISMS OF CERTAIN FLAG MANIFOLDS

Duan Haibao and Zhao Xuan 


\title{
THE CLASSIFICATION OF COHOMOLOGY ENDOMORPHISMS OF CERTAIN FLAG MANIFOLDS
}

\author{
DUAN HaibaO AND ZHAO XUAN
}

This note describe a method for dertermining cohomology endomorphisms of a space. Examples are taken for some flag manifolds.

Let $U(n)$ be the unitary group of rank $n, S O(m)$ the special orthogonal group of rank $m$, and $S p(n)$, the symplectic group of rank $n$. Fix, once and for all, a maximal torus $T^{n} \subset U(n), T^{\left[\frac{m}{2}\right]} \subset S O(m), T^{n} \subset S p(n)$ in each of the groups.

For a topological space $X$ the problem of finding all integral cohomology endomorphisms $H^{*}(X ; Z) \rightarrow H^{*}(X ; Z)$ is a step toward classifying all homotopy classes of self maps $X \rightarrow X$. In [3] M. Hoffman solved this problem for the flag manifold $F(n)=U(n) / T^{n}$. In this note we settle the problem for the spaces $D(m)=S O(m) / T^{\left[\frac{m}{2}\right]}$ and $S(n)=S p(n) / T^{n}$.

It is worth to point out that S. Papadima determined all cohomology automorphisms of $G / T$ with coefficients in $R$ or $Q$, where $G$ is a compact connected Lie Group and $T$ its maximal torus [5].

2 .

We describe the integral cohomologies (in terms of generators-relations) of these spaces. Let $e_{i}\left(z_{1}, \cdots, z_{n}\right)$ be the $i^{\text {th }}$ elementary symmetric function in the variables $z_{1}, \cdots, z_{n}$. The following results is well known from A. Borel $[\mathbf{1}]$.

\section{Lemma 1.}

$$
\begin{aligned}
& H^{*}(F(n) ; Z)=Z\left[t_{1}, \ldots, t_{n}\right] / e_{i}\left(t_{1}, \ldots, t_{n}\right), 1 \leq i \leq n,\left|t_{i}\right|=2 ; \\
& H^{*}(S(n) ; Z)=Z\left[y_{1}, \ldots, y_{n}\right] / e_{i}\left(y_{1}^{2}, \ldots, y_{n}^{2}\right), 1 \leq i \leq n,\left|y_{i}\right|=2,
\end{aligned}
$$

and there exist integral classes $x_{1}, \ldots, x_{n} \in H^{2}(D(m) ; Z), n=\left[\frac{m}{2}\right]$, so that

$$
\begin{gathered}
H^{*}(D(2 n) ; Q)=Q\left[x_{1}, \ldots, x_{n}\right] / e_{i}\left(x_{1}^{2}, \ldots, x_{n}^{2}\right), \\
1 \leq i \leq n-1 ; e_{n}\left(x_{1}, \ldots, x_{n}\right) ; \\
H^{*}(D(2 n+1) ; Q)=Q\left[x_{1}, \ldots, x_{n}\right] / e_{i}\left(x_{1}^{2}, \ldots, x_{n}^{2}\right), 1 \leq i \leq n,
\end{gathered}
$$


where $|u|$ stands for the grade of the enclosed $u$ and $Q$, the field of rationals.

The integral cohomology for the space $D(m)$ is not available in [1] due to the presence of 2 -torsion in $H^{*}(S O(m) ; Z)$. However, with slightly more geometric investigation, the algebra $H^{*}(D(m) ; Z)$ can be shown to be as follows.

Let $G(2 n, 2)$ be the Grassmannian of oriented 2-planes through the origin in the Euclidean $2 n$-space $R^{2 n}$. The canonical 2-plane bundle $\gamma$ over $G(2 n, 2)$ will have the preferred orientation. Let $x \in H^{2}(G(2 n, 2) ; Z)$ be the Euler classes for the oriented bundle $\gamma$.

The space $D(2 n)$ can be identified with the subspace of $G(2 n, 2) \times \cdots \times$ $G(2 n, 2)$ (n-copies) consisting of $n$ mutually orthogonal oriented 2-planes with their induced orientation on $R^{2 n}$ agrees with the standard one. Evidently we have $n$ projections $\pi_{i}: D(2 n) \rightarrow G(2 n, 2), 1 \leq i \leq n$, given by taking the $i^{\text {th }}$ plane. Set $x_{i}=\pi_{i}^{*}(x) \in H^{2}(D(2 n) ; Z)$.

Lemma 2. The integral classes $e_{i}\left(x_{1}, \ldots, x_{n}\right) \in H^{*}(D(2 n) ; Z), 1 \leq i \leq n$, are all divisible by 2 . Further the algebra $H^{*}(D(2 n) ; Z)$ is generated multiplicatively by $x_{1}, \ldots, x_{n-1}$ and $v=\frac{1}{2} e_{1}\left(x_{1}, \ldots, x_{n}\right)$ subject to the relations

$$
\frac{1}{4} e_{i}\left(x_{1}^{2}, \ldots, x_{n}^{2}\right)=0,1 \leq i \leq n-1, \text { and } \frac{1}{2} e_{n}\left(x_{1}, \ldots, x_{n}\right)=0,
$$

where the 4-divisibility of $e_{i}\left(x_{1}^{2}, \ldots, x_{n}^{2}\right)$ follows from the 2-divisibility of $e_{i}\left(x_{1}, \ldots, x_{n}\right)$.

From the Wang sequence of the standard fibration

$$
D(2 n) \subset D(2 n+1) \stackrel{p}{\rightarrow} S^{2 n}
$$

we find classes $x_{1}^{\prime}, \ldots, x_{n}^{\prime} \in H^{2}(D(2 n+1) ; Z)$ so that $x_{i}^{\prime} \mid D(2 n)=x_{i} \in$ $H^{2}(D(2 n) ; Z)$, i.e., the bundle $p$ has Leray-Hirsch property [6, p. 365]. Moreover it can be shown that $p^{*}(\iota)= \pm \frac{1}{2} x_{1}^{\prime} \cdots x_{n}^{\prime}$, where $\iota \in H^{2 n}\left(S^{2 n} ; Z\right)=$ $Z$ is a generator and $p^{*}$, the induced homomorphism. Therefore, Lemma 2 implies:

Lemma 3. The integral classes $e_{i}\left(x_{1}^{\prime}, \ldots, x_{n}^{\prime}\right) \in H^{*}(D(2 n+1) ; Z), 1 \leq i \leq$ $n$, are all divisible by 2 . Further the algebra $H^{*}(D(2 n+1) ; Z)$ is generated multiplicatively by $x_{1}^{\prime}, \ldots, x_{n-1}^{\prime}$ and $v^{\prime}=\frac{1}{2} e_{1}\left(x_{1}^{\prime}, \ldots, x_{n}^{\prime}\right)$ subject to the relations

$$
\frac{1}{4} e_{i}\left(x_{1}^{\prime 2}, \ldots, x_{n}^{\prime 2}\right)=0, \quad 1 \leq i \leq n .
$$

3.

Let $X$ denote one of the spaces $F(n), D(m)$, or $S(n)$. From the descriptions for $H^{*}(X ; Z)$ two types of endomorphisms become apparent:

Example 1. For an integer $k \in Z$, the Adams map $l_{k}: H^{*}(X ; Z) \rightarrow$ $H^{*}(X ; Z)$ is defined by $l_{k}(u)=k u$ for all $u \in H^{2}(X ; Z)$. 
Example 2. Let $\Sigma_{n}$ be the permutation group on $\{1,2, \ldots, n\}$, and let $S_{n}$ be the full permutation group on $\{ \pm 1, \pm 2, \ldots, \pm n\}$. We set

$$
\Phi(X)= \begin{cases}\Sigma_{n} & \text { if } X=F(n) \text { and } \\ S_{n} & \text { if } X=D(2 n), D(2 n+1) \text { or } S(n) .\end{cases}
$$

For a $\alpha \in \Phi(X)$ the standard action of $\alpha$ on $H^{2}(X ; Z)$ extends uniquely to an algebra map $H^{*}(X ; Z) \rightarrow H^{*}(X ; Z)$, for which we denote still by $\alpha$.

For an $k \in Z$ and a $\alpha \in \Phi(X)$ write $h_{k}^{\alpha}$ for the composition $\alpha \circ l_{k}$. In [3] M. Hoffman proved that any endomorphism of $H^{*}(F(n) ; Z)$ has the form $h_{k}^{\alpha}$ for some $k \in Z$ and $\alpha \in \Phi(F(n))$. We extends his result in:

Theorem 1. For $X=D(m), m>5, m \neq 8$ or $S(n), n>2$, any endomorphism of $H^{*}(X ; Z)$ has the form $h_{k}^{\alpha}$ for some $k \in Z$ and $\alpha \in \Phi(X)$.

Exceptions do occur for the spaces $D(4), D(5), D(8)$ and $S(2)$. Indeed, as only small values of $n$ are involved, a direct computation based on Lemma 1-3 yields the following. Let $h$ be an endomorphism of $H^{*}(X ; Z)$ with $X=$ $D(4), D(5), D(8)$ or $S(2)$. With respect to the $Q$-basis of $H^{2}(X ; Z)$ given in Lemma 1 the action of $h$ on $H^{2}(X ; Z)$ has the representations:

1) $h_{k}^{\alpha}$, or $\left(\begin{array}{cc}-1 & 1 \\ 1 & 1\end{array}\right) \circ h_{k}^{\alpha}$, with $k \in Z, \alpha \in S_{2}$ for $X=S(2)$;

2) $\frac{1}{2}\left(\begin{array}{cc}a & \epsilon a \\ b & \epsilon b\end{array}\right)$, or $\left(\begin{array}{cc}a & \epsilon b \\ b & \epsilon a\end{array}\right)$, with $a, b \in Z, \epsilon= \pm 1$ for $X=D(4)$;

3) $h_{k}^{\alpha}$, or $\frac{1}{2}\left(\begin{array}{cc}-1 & 1 \\ 1 & 1\end{array}\right) \circ h_{k}^{\alpha}$, with $k \in Z, \alpha \in S_{2}$ for $X=D(5)$ and

4) $h_{k}^{\alpha}$, or $\frac{1}{2}\left(\begin{array}{cccc}-1 & 1 & 1 & 1 \\ 1 & -1 & 1 & 1 \\ 1 & 1 & -1 & 1 \\ 1 & 1 & 1 & -1\end{array}\right) \circ h_{k}^{\alpha}$, with $k \in Z, \alpha \in S_{4}$ for $X=D(8)$.

4.

In studying endomorphisms of an algebra, a technique of handling relations appears to be crucial. The proof of Theorem 1 serves the purpose of introducing the so called "quantification" method. By this we mean polynomial relations might be quantified, hence simplified, by evaluating them at appropriate quantities. More precisely, the proof of Theorem 1 will be based on the following numerical results.

For a complex number $y \in C$ denote respectively by $\langle y\rangle$, and $[y]$ for the sets of sequences

$$
\begin{aligned}
& \left\{\left(\epsilon_{1}, \epsilon_{2} y, \ldots, \epsilon_{n} y^{n-1}\right)_{\alpha} \mid \alpha \in \Sigma_{n}, \epsilon_{i}= \pm 1\right\} ; \\
& \left\{\left(0, \epsilon_{1}, \epsilon_{2} y, \ldots, \epsilon_{n-1} y^{n-2}\right)_{\alpha} \mid \alpha \in \Sigma_{n}, \epsilon_{i}= \pm 1\right\},
\end{aligned}
$$

where, for instance, $\left(z_{1}, \ldots, z_{n}\right)_{\alpha}=\left(z_{\alpha(1)}, \ldots, z_{\alpha(n)}\right)$. 
Theorem 2. Let $a_{1}, \ldots, a_{n} ; r$ be some reals, and let $\xi=e^{\frac{\pi i}{n}}, \eta=e^{\frac{\pi i}{n-1}}$.

(1) If $n>2$ and if the equality $\left(a_{1} x_{1}+\cdots+a_{n} x_{n}\right)^{2 n}=r$ holds for all $\left(x_{1}, \ldots, x_{n}\right) \in\langle\xi\rangle$, then at most one of $a_{1}, \ldots, a_{n}$ is non-zero.

(2) If $n>4$ and if the equality $\left(a_{1} x_{1}+\cdots+a_{n} x_{n}\right)^{2 n}=r\left(a_{1} x_{1}+\cdots+a_{n} x_{n}\right)^{2}$ holds for all $\left(x_{1}, \ldots, x_{n}\right) \in[\eta]$, then at most one of $a_{1}, \ldots, a_{n}$ is nonzero.

\section{5.}

The proof of Theorem 2 will be postponed until the next section and at this moment, we show how it leads to a proof of Theorem 1 . Let $h$ be an endomorphism of $H^{*}(X ; Z)$. Define a matrix $A=\left(a_{i j}\right)_{n \times n}$ by

$$
h\left(y_{i}\right)=\Sigma_{j} a_{i j} y_{j}, a_{i j} \in Z, \quad \text { for } X=S(n) ;
$$

(resp. $h\left(x_{i}\right)=\Sigma_{j} a_{i j} x_{j}, a_{i j} \in Q$, for $X=D(m)$ with $m=2 n$ or $\left.2 n+1\right)$.

Lemma 4. If $n>2$ (resp. $m>5$ and $m \neq 8$ ), at most one entry in each row of $A$ is nonzero.

Proof. Consider first the case $X=S(n), n>2$ (resp. $D(2 n+1), n>2$; for rationally the cohomology of $S(n)$ is isomorphic to that of $D(2 n+1)$ by Lemma 1). The obvious formula in $Z\left[y_{1}, \ldots, y_{n}\right]$

$$
\begin{aligned}
y_{i}^{2 n}= & y_{i}^{2(n-1)} e_{1}\left(y_{1}^{2}, \ldots, y_{n}^{2}\right)-y_{i}^{2(n-2)} e_{2}\left(y_{1}^{2}, \ldots, y_{n}^{2}\right) \\
& +\cdots+(-1)^{n-1} e_{n}\left(y_{1}^{2}, \ldots, y_{n}^{2}\right)
\end{aligned}
$$

implies, in $H^{*}(S(n) ; Z)$, that $y_{i}^{2 n}=0$, and consequently $h\left(y_{i}\right)^{2 n}=0$. Thus in $Z\left[y_{1}, \ldots, y_{n}\right]$ we have

$h\left(y_{i}\right)^{2 n}=\left(a_{i 1} y_{1}+\cdots+a_{i n} y_{n}\right)^{2 n}=g_{1} e_{1}\left(y_{1}^{2}, \ldots, y_{n}^{2}\right)+\cdots+g_{n} e_{n}\left(y_{1}^{2}, \ldots, y_{n}^{2}\right)$,

for some $g_{i} \in Z\left[y_{1}, \ldots, y_{n}\right], 1 \leq i \leq n$, with $\left|g_{i}\right|=4(n-i)$. Note that $g_{n}=r$ $\in Z$ for degree reason. Since the systems

$$
e_{i}\left(y_{1}^{2}, \ldots, y_{n}^{2}\right)=0,1 \leq i \leq n-1 ; \quad e_{n}\left(y_{1}^{2}, \ldots, y_{n}^{2}\right)=1
$$

is satisfied by all $\left(y_{1}, \ldots, y_{n}\right) \in\langle\xi\rangle, \xi=e^{\frac{\pi i}{n}}$, we get

$$
\left(a_{i 1} y_{1}+\cdots+a_{i n} y_{n}\right)^{2 n}=r \text { for all }\left(y_{1}, \ldots, y_{n}\right) \in\langle\xi\rangle .
$$

So $h\left(y_{i}\right)=a y_{j}$ for some $a \in Z, 1 \leq j \leq n$, by 1$)$ of Theorem 2 .

Consider next $X=D(2 n), n>4$. Applying $h$ to the standard equality

$$
\begin{aligned}
x_{i}^{2 n}= & x_{i}^{2(n-1)} e_{1}\left(x_{1}^{2}, \ldots, x_{n}^{2}\right)-x_{i}^{2(n-2)} e_{2}\left(x_{1}^{2}, \ldots, x_{n}^{2}\right) \\
& +\cdots+(-1)^{n-1} e_{n}\left(x_{1}, \ldots, x_{n}\right)^{2}
\end{aligned}
$$


in $Q\left[x_{1}, \ldots, x_{n}\right]$ and using (since $h$ preserves the ideal) the facts

$$
\begin{aligned}
h\left(e_{i}\left(x_{1}^{2}, \ldots, x_{n}^{2}\right)\right) & =\Sigma_{j \leq i} g_{i j} e_{j}\left(x_{1}^{2}, \ldots, x_{n}^{2}\right)+l_{i} e_{n}\left(x_{1}, \ldots, x_{n}\right) ; \\
h\left(e_{n}\left(x_{1}, \ldots, x_{n}\right)\right) & =\Sigma_{2 i<n} k_{i} e_{i}\left(x_{1}^{2}, \ldots, x_{n}^{2}\right)+l e_{n}\left(x_{1}, \ldots, x_{n}\right)
\end{aligned}
$$

we find

$$
\begin{array}{r}
h\left(x_{i}\right)^{2 n}=f \in\left\{\text { the ideal generated by } e_{i}\left(x_{1}^{2}, \ldots, x_{n}^{2}\right), 1 \leq i \leq n-2 ;\right. \\
\left.\qquad e_{n}\left(x_{1}, \ldots, x_{n}\right)\right\}+g_{n-1, n-1} h\left(x_{i}\right)^{2} e_{n-1}\left(x_{1}^{2}, \ldots, x_{n}^{2}\right),
\end{array}
$$

where $g_{n-1, n-1}=r \in Q$ for degree reasons. Since the systems

$$
e_{n}\left(x_{1}, \ldots, x_{n}\right)=e_{i}\left(x_{1}^{2}, \ldots, x_{n}^{2}\right)=0,1 \leq i \leq n-2 ; e_{n-1}\left(x_{1}^{2}, \ldots, x_{n}^{2}\right)=1
$$

is satisfied by all $\left(x_{1}, \ldots, x_{n}\right) \in[\eta]$ we get

$$
h\left(x_{i}\right)^{2 n}=r h\left(x_{i}\right)^{2} \text { for all }\left(x_{1}, \ldots, x_{n}\right) \in[\eta] .
$$

Thus $h\left(x_{i}\right)=a x_{j}$ for some $a \in Q, 1 \leq j \leq n$, by 2$)$ of Theorem 2 .

The case $X=D(6)$ requires additional treatment as assertion 2) of Theorem 2 does not apply to it. Assume, with respect to the $Q$-basis $x_{1}, x_{2}, x_{3} \in H^{2}(D(6) ; Z)$, that $h$ has the matrix representation

$$
A=\left(\begin{array}{ccc}
a_{1} & a_{2} & a_{3} \\
b_{1} & b_{2} & b_{3} \\
c_{1} & c_{2} & c_{3}
\end{array}\right), a_{i}, b_{j}, c_{k} \in Q .
$$

Since $h$ preserves the ideal, we have

$$
\begin{aligned}
& h\left(x_{1}^{2}+x_{2}^{2}+x_{3}^{2}\right)=l\left(x_{1}^{2}+x_{2}^{2}+x_{3}^{2}\right) ; \quad \text { and } \\
& h\left(x_{1} x_{2} x_{3}\right)=g\left(x_{1}^{2}+x_{2}^{2}+x_{3}^{2}\right)+k x_{1} x_{2} x_{3}
\end{aligned}
$$

for some $l, k \in Q, g \in Q\left[x_{1}, x_{2}, x_{3}\right]$. From (5.1) we get $\Sigma a_{i}^{2}=\Sigma b_{i}^{2}=\Sigma c_{i}^{2}=l$. If $l=0$ we are done, so we may assume that $l \neq 0$ (thus $A$ has no zero row). Putting $\left(x_{1}, x_{2}, x_{3}\right)=(0, i t, t), i=\sqrt{-1}, t \in R$, in (5.2) gives

$$
\left(a_{2} i+a_{3}\right)\left(b_{2} i+b_{3}\right)\left(c_{2} i+c_{3}\right) t^{3}=0 \text { for all } t \in R .
$$

So one of the three complex numbers $\left(a_{2} i+a_{3}\right),\left(b_{2} i+b_{3}\right),\left(c_{2} i+c_{3}\right)$ must be zero. Assume, without losing the generality, that $a_{2}=a_{3}=0$ (hence $\left.a_{1} \neq 0\right)$. Then (5.2) becomes

$$
a_{1} x_{1}\left(\Sigma b_{i} x_{i}\right)\left(\Sigma c_{i} x_{i}\right)=g\left(x_{1}^{2}+x_{2}^{2}+x_{3}^{2}\right)+k x_{1} x_{2} x_{3} .
$$

Setting $\left(x_{1}, x_{2}, x_{3}\right)=(t, i t, 0)$ we get $a_{1}\left(b_{1}+b_{2} i\right)\left(c_{1}+c_{2} i\right) t^{3}=0$ for all $t \in R$. So we may assume further that $b_{1}=b_{2}=0, b_{3} \neq 0$. Finally from

$$
a_{1} b_{3} x_{1} x_{3}\left(\Sigma c_{i} x_{i}\right)=g\left(x_{1}^{2}+x_{2}^{2}+x_{3}^{2}\right)+k x_{1} x_{2} x_{3}
$$

we get $c_{1}=c_{3}=0$. This completes the proof of Lemma 4 . 
Proof of Theorem 1.

Case 1. $X=S(n), n \geq 3$. Since $h$ must preserve the ideal we have, in particular, that $h\left(y_{1}^{2}+\cdots+y_{n}^{2}\right)=l\left(y_{1}^{2}+\cdots+y_{n}^{2}\right)$ for some $l \in Z$. Now $h\left(y_{1}^{2}+\cdots+y_{n}^{2}\right)=\Sigma_{j}\left(\Sigma_{i} a_{i j}^{2}\right) y_{j}^{2}$ (by Lemma 4) tells that

$$
\text { all column sum of }\left(a_{i j}^{2}\right)_{n \times n} \text { are equal (to } l \text { ). }
$$

If $l=0$, then $A=0_{n \times n}$, we are clearly done. If $l \neq 0$ then (5.3) indicates that $A$ has no zero column. A combination of Lemma 4 and (5.3) now gives that $A=k P$, where $k \in Z$ with $k^{2}=l$, and where $P$ is the matrix representation of some $\alpha \in S_{n}$. This completes the proof for $X=S(n)$.

Case 2. $\quad X=D(m), m \geq 6$ and $m \neq 8$. A discussion similar to Case 1 shows that $h=h_{k}^{\alpha}$ for some $k \in Q, \alpha \in S_{n}$. Further, as $h$ must send integral classes to integral ones we have $k \in Z$ by Lemma 2 and 3 . This finishes the proof of Theorem 1.

\section{Proof of Theorem 2.}

Assume throughout this section that $a_{1}, \ldots, a_{n} ; r$ are some real numbers satisfying

$$
\begin{aligned}
& \left(a_{1} x_{1}+\cdots+a_{n} x_{n}\right)^{2 n} \\
& =r \text { for all }\left(x_{1}, \ldots, x_{n}\right) \in\langle\xi\rangle \\
& \left(\operatorname{resp} .\left(a_{1} x_{1}+\cdots+a_{n} x_{n}\right)^{2 n}\right. \\
& \left.=r\left(a_{1} x_{1}+\cdots+a_{n} x_{n}\right)^{2} \text { for all }\left(x_{1}, \ldots, x_{n}\right) \in[\eta]\right)
\end{aligned}
$$

where $\xi=e^{\frac{\pi}{n}}$ (resp. $\left.\eta=e^{\frac{\pi}{n-1}}\right)$. Clearly we can also assume that $a_{i} \geq 0$ for all $1 \leq i \leq n$ (by replacing $a_{i}$ with $\operatorname{sign}\left(a_{i}\right) a_{i}$ when $a_{i}<0$ ). If $r=0$, Theorem 2 is obviously true. So we may adopt the convention that $r \neq 0$.

Denote by $C^{+}$the upper half complex plane $\{a+b i \mid a, b \in R, b \geq 0\}$, and write

$$
\begin{aligned}
H_{1}=\left\{\omega_{\alpha}=a_{\alpha(1)}+a_{\alpha(2)} \xi+\cdots+a_{\alpha(n)} \xi^{n-1} \mid \alpha \in \Sigma_{n}\right\} & \\
\left(\text { resp. } H_{2}=\left\{\omega_{\alpha}^{\prime}=a_{\alpha(1)}+\right.\right. & a_{\alpha(2)} \eta \\
& \left.\left.+\cdots+a_{\alpha(n-1)} \eta^{n-2}+a_{\alpha(n)} \cdot 0 \mid \alpha \in \Sigma_{n}\right\}\right) .
\end{aligned}
$$

Since $\xi^{k} \in C^{+}$for all $0 \leq k \leq n-1$ (resp. $\eta^{l} \in C^{+}$for $0 \leq l \leq n-2$ ), and since $a_{i} \geq 0$, the set $H_{1}$ (resp. $H_{2}$ ) of complex numbers can be considered as a subset of $C^{+}$. We observe first of all that:

Lemma 5. If the sequence $a_{1}, \ldots, a_{n}, n \geq 3$, contains more than one nonzero entry then $\# H_{i} \leq n-1, i=1,2$. 
Proof. Let $\varphi$ be the polar angle function (with respect to the oriented real axis) on nonzero complexes. Take a base point $\omega_{0} \in H_{1}$ (resp. $\omega_{0}^{\prime} \in H_{2}$ ) with

$$
\begin{aligned}
& \varphi\left(\omega_{0}\right)=\min \left\{\varphi\left(\omega_{\alpha}\right) \mid \omega_{\alpha} \in H_{1} \backslash 0\right\} \\
& \text { (resp. } \left.\varphi\left(\omega_{0}^{\prime}\right)=\min \left\{\varphi\left(\omega_{\alpha}^{\prime}\right) \mid \omega_{\alpha}^{\prime} \in H_{2} \backslash 0\right\}\right) .
\end{aligned}
$$

Clearly if the sequence $a_{1}, \cdots, a_{n}$ contains more than one nonzero entry then we have $0 \notin H_{i}$, and for all $\omega_{\alpha} \in H_{1}$ (resp. $\omega_{\alpha}^{\prime} \in H_{2}$ )

$$
\begin{aligned}
& 0<\varphi\left(\omega_{0}\right) \leq \varphi\left(\omega_{\alpha}\right)<\frac{n-1}{n} \pi \\
& \left(\text { resp. } 0 \leq \varphi\left(\omega_{0}^{\prime}\right) \leq \varphi\left(\omega_{\alpha}^{\prime}\right) \leq \frac{n-2}{n-1} \pi\right) .
\end{aligned}
$$

This indicates that, since $\omega_{\alpha}^{2 n}=r\left(\right.$ resp. $\left.\left(\omega_{\alpha}^{\prime}\right)^{2 n}=r\left(\omega_{\alpha}^{\prime}\right)^{2}\right)$, for each $\alpha \in \Sigma_{n}$ there exists an integer $0 \leq k_{\alpha} \leq n-2$ (resp. $0 \leq l_{\alpha} \leq n-2$ ) so that $\omega_{\alpha}=\xi^{k_{\alpha}} \omega_{0}\left(\right.$ resp. $\left.\omega_{\alpha}^{\prime}=\eta^{l_{\alpha}} \omega_{0}^{\prime}\right)$. This verifies \# $H_{1}\left(\right.$ resp. $\left.\# H_{2}\right) \leq n-1$.

On the other hand a common lower bound for $\# H_{i}$ can be obtained as follows. Since both sets $H_{i}$ are invariant under the given action of $\Sigma_{n}$ we can assume, without loss the generality, that $a_{1} \geq a_{2} \geq \cdots \geq a_{n}$. Thus a partition $r_{1}, \cdots, r_{k}$ for $n$ (i.e., $r_{i} \geq 1, \Sigma r_{i}=n$ ) can be found so that, if $s_{h}=r_{1}+\cdots+r_{h}$ then

$$
a_{s_{h}+1}=\cdots=a_{s_{h+1}}, \quad a_{s_{h}+1}>a_{s_{h}} \quad \text { for all } h=1, \ldots, k .
$$

For a pair of integers $1 \leq i<j \leq n$ let $(i, j) \in \Sigma_{n}$ be the transposition of $i$ and $j$. It is easy to see that the cardinality of the set

$$
\bar{\Sigma}_{n}=\left\{\alpha_{0},(i, j) \mid i \in\left[s_{h}+1, s_{h+1}\right], j \in\left[s_{h^{\prime}}+1, s_{h^{\prime}+1}\right], 1 \leq h<h^{\prime} \leq k\right\}
$$

is $1+\Sigma_{1 \leq i<j \leq k} r_{i} r_{j}$. However for a $\alpha=(i, j)$ we have

$$
\begin{gathered}
\omega_{\alpha}=\omega_{i d}+\left(a_{i}-a_{j}\right)\left(\xi^{j-1}-\xi^{i-1}\right) \in H_{1} \\
\left(\begin{array}{ll}
\text { resp. } \omega_{\alpha}^{\prime} & =\omega_{i d}^{\prime}+\left\{\begin{array}{ll}
\left(a_{i}-a_{j}\right)\left(\eta^{j-1}-\eta^{i-1}\right) & \text { if } j<n \\
\left(a_{n}-a_{i}\right) \eta^{i-1} & \text { if } j=n
\end{array} \in H_{2}\right.
\end{array}\right),
\end{gathered}
$$

where $i d \in S_{n}$ is the identity. We use this to show:

Lemma 6. Assume as above. Then $\# H_{i} \geq 1+\Sigma_{1 \leq i<j \leq k} r_{i} r_{j}$.

Proof. It suffices to show that if $\alpha, \beta \in \bar{\Sigma}_{n}$ with $\alpha \neq \beta$, then $\omega_{\alpha} \neq \omega_{\beta}$ (resp. $\left.\omega_{\alpha}^{\prime} \neq \omega_{\beta}^{\prime}\right)$. We verify the first assertion for instance. If one of $\alpha, \beta$ is the identity, it is clearly true. Assume $\alpha=(i, j), \beta=(i \prime, j \prime) \in \bar{\Sigma}_{n}$ are such that $\omega_{\alpha}=\omega_{\beta}$. Then $\left(a_{i}-a_{j}\right)\left(\xi^{j-1}-\xi^{i-1}\right)=\left(a_{i^{\prime}}-a_{j^{\prime}}\right)\left(\xi^{j^{\prime}-1}-\xi^{i^{\prime}-1}\right)$ by $(6.1)$. 
Comparing the real and imaginary parts gives

$$
\left(a_{i}-a_{j}\right) \sin \frac{j-i}{2 n} \pi \sin \frac{i+j-2}{2 n} \pi=\left(a_{i^{\prime}}-a_{j^{\prime}}\right) \sin \frac{j^{\prime}-i^{\prime}}{2 n} \pi \sin \frac{i^{\prime}+j^{\prime}-2}{2 n} \pi ;
$$

$$
\left(a_{i}-a_{j}\right) \sin \frac{j-i}{2 n} \pi \cos \frac{i+j-2}{2 n} \pi=\left(a_{i^{\prime}}-a_{j^{\prime}}\right) \sin \frac{j^{\prime}-i^{\prime}}{2 n} \pi \cos \frac{i^{\prime}+j^{\prime}-2}{2 n} \pi .
$$

Taking the quotient of (6.2) by (6.3) yields $t g \frac{i+j-2}{2 n} \pi=t g \frac{i^{\prime}+j^{\prime}-2}{2 n} \pi$. Since $1 \leq i<j \leq n, 1 \leq i^{\prime}<j^{\prime} \leq n$, the monodromy of $t g$ on $[0, \pi]$ tells

$$
i+j=i \prime+j \prime \text {. }
$$

Now (6.2) gives

$$
\left(a_{i}-a_{j}\right) \sin \frac{j-i}{2 n} \pi=\left(a_{i^{\prime}}-a_{j^{\prime}}\right) \sin \frac{j^{\prime}-i^{\prime}}{2 n} \pi .
$$

We may assume that $i \leq i^{\prime}$. (6.4) then implies $i \leq i^{\prime}<j^{\prime} \leq j$. So we have $\left(a_{i}-a_{j}\right) \geq\left(a_{i^{\prime}}-a_{j^{\prime}}\right)(>0)$ and that

$$
\sin \frac{j-i}{2 n} \geq \sin \frac{j^{\prime}-i^{\prime}}{2 n}
$$

while the equality holds if and only if $j-i=j^{\prime}-i^{\prime}$

(by the monotonicity of $\sin$ on $\left.\left[0, \frac{\pi}{2}\right]\right)$. Now $(6.5)$ says that

$$
j-i=j^{\prime}-i^{\prime} \text {. }
$$

Summarizing $\omega_{\alpha}=\omega_{\beta}$ (resp. $\omega_{\alpha}^{\prime}=\omega_{\beta}^{\prime}$ ), $\alpha, \beta \in \bar{\Sigma}_{n}$, will imply $\alpha=\beta$ by (6.4) and (6.7). This completes the proof of Lemma 6.

Since

$$
\begin{aligned}
& 1+\Sigma_{1 \leq i<j \leq k} r_{i} r_{j} \\
& =1+\left(n-r_{k}\right) r_{k}+\Sigma_{1 \leq i<j \leq k-1} r_{i} r_{j}\left(\text { since } r_{1}+\cdots+r_{k}=n\right) \\
& =n+\left(n-r_{k}-1\right)\left(r_{k}-1\right)+\Sigma_{1 \leq i<j \leq k-1} r_{i} r_{j} \\
& \begin{cases}>n & \text { if } k \geq 3 \\
=n+\left(r_{1}-1\right)\left(r_{2}-1\right) & \text { if } k=2 \\
=1 & \text { if } k=1\end{cases}
\end{aligned}
$$

a combination of Lemma 5 and 6 gives:

Lemma 7. If the sequence $a_{1}, \ldots, a_{n}, n \geq 3$, contains more than one nonzero entry, then $a_{1}=\cdots=a_{n}=b$ for some $b \neq 0$.

We complete the proof of Theorem 2 by showing:

Lemma 8. If $a_{1}=\cdots=a_{n}=b$ for some $b \neq 0$, then $n \leq 2($ resp. $n \leq 4)$. 
Proof. Here we have $H_{1}=\left\{\omega_{0}=\frac{2 b}{1-\xi}\right\}$ (resp. $H_{2}=\left\{\omega_{0}^{\prime}=\frac{2 b}{1-\eta}\right\}$ ). We put

$$
\begin{aligned}
& J_{1}=\left\{\omega_{k}=\omega_{0}-2 b \xi^{k-1} \mid k=1, \ldots, n\right\} \\
& \text { (resp. } J_{2}=\left\{\omega_{k}^{\prime}=\omega_{0}^{\prime}-2 b \eta^{k-1} \mid k=1, \ldots, n-1\right\} \text { ). }
\end{aligned}
$$

It suffices to show that

$$
\left.\# J_{1}=n \leq 2 \text { (resp. } \# J_{2}=n-1 \leq 3\right) .
$$

Let $S_{1}$ be the circle in the complex plane centered at $\omega_{0}\left(\right.$ resp. $\left.\omega_{0}^{\prime}\right)$ with radius $2|b|$. Then $J_{i} \subset S_{1}, i=1,2$. On the other hand, if we let $S_{2}$ be the circle centered at 0 with radius $\sqrt{|r|^{\frac{1}{2 n}}}\left(\operatorname{resp} \cdot \sqrt{|r|^{\frac{1}{2 n-2}}}\right)$, then we have $J_{1} \subset S_{2}$ since $\left(\omega_{k}\right)^{2 n}=r$ for all $1 \leq k \leq n$ (resp. $J_{2} \subset S_{2} \cup 0$ since $\left(\omega_{k}^{\prime}\right)^{2 n}=r\left(\omega_{k}^{\prime}\right)^{2}$ for all $\left.1 \leq k \leq n-1\right)$. (6.8) is now verified by

$$
\left.J_{1} \subseteq S_{1} \cap S_{2} \text { (resp. } J_{2} \subseteq S_{1} \cap\left(S_{2} \cup 0\right)\right) .
$$

Remark 1. The following result, on which the main results of $[\mathbf{2}]$ and [3] are based, was first proved in [4] and a different proof was given in [2].

"If $u \in H^{2}(F(n) ; Z)$ and $u^{n}=0$, then $u=a t_{i}$ for some $a \in Z, 1 \leq i \leq n$." Our proof of Lemma 4 indicates that its analogue holds also for the spaces $S(n)$ and $D(2 n+1)$.

Remark 2. One may find the following corollary of Lemma 7 useful in verifying the assertion made in Section 3, exceptional Case 4.

"Let $a_{1}, \cdots, a_{4} ; r$ be some reals, and let $\eta=e^{\frac{\pi i}{3}}$. If the equality $\left(a_{1} x_{1}+\right.$ $\left.\cdots+a_{4} x_{4}\right)^{8}=r\left(a_{1} x_{1}+\cdots+a_{4} x_{4}\right)^{2}$ holds for all $\left(x_{1}, \ldots, x_{4}\right) \in[\eta]$, then we have either 1) at most one of $a_{1}, \ldots, a_{n}$ is non-zero or, 2) $\left|a_{1}\right|=\cdots=\left|a_{4}\right|$." Instead, a proof based on Lemma 2 will cause tedious computation.

Acknowledgement. The authors feel grateful to Boju Jiang. The present proof of Lemma 8, more intuitive and shorter than the previous one, is due to him.

\section{References}

[1] A. Borel, Sur la cohomologie des espaces fibres principaux et des espaces homogenes de groupes de Lie compacts, Ann. of Math., 57 (1953), 115-207.

[2] J. Ewing and A. Liulevicius, Homotopy rigidity of linear actions on homogeneous spaces, J. Pure and Applied Algebra, 18 (1990), 259-267.

[3] M. Hoffman, On fixed point free maps of the complex flag manifold, Indiana U. Math. J., 33 (1984), 249-255.

[4] J.D. Monk, The geometry of flag manifolds, Proc. London Math. Soc., 9(3) (1959), 253-286. 
[5] S. Papadima, Rigidity properties of compact Lie group module maximal tori, Math. Ann., 275 (1986) 637-652.

[6] R.M. Switzer, Algebraic topology-homotopy and homology, Springer-Verlag BerlinHeidelberg, 1975.

Received October 22, 1997 and revised October 20, 1998. Project 19771001 supported by NSFC.

PEKING UNIVERSITY

BeIJING 100871

P. R. CHINA

E-mail address: dhb@sxx0.math.pku.edu.cn

Peking University

BEIJING 100871

P. R. China 